\title{
Numerical and the MU radar estimations of gravity wave enhancement and turbulent ozone fluxes near the tropopause
}

\author{
N. M. Gavrilov ${ }^{1}$ and S. Fukao ${ }^{2}$ \\ ${ }^{1}$ Saint-Petersburg State University, Atmospheric Physics Department, Petrodvorets, 198 504, St. Petersburg, Russia \\ ${ }^{2}$ Kyoto University, Center for Atmospheric and Space Research, Uji, Kyoto 611, Japan
}

Received: 2 December 2003 - Revised: 18 June 2004 - Accepted: 24 June 2004 - Published: 29 November 2004

Part of Special Issue "10th International Workshop on Technical and Scientific Aspects of MST Radar (MST10)"

\begin{abstract}
It is shown with a numerical simulation that a sharp increase in the vertical temperature gradient and BruntVäisälä frequency near the tropopause may produce an increase in the amplitudes of internal gravity waves (IGWs) propagating upward from the troposphere, wave breaking and generation of stronger turbulence. This may enhance the transport of admixtures between the troposphere and stratosphere in the middle latitudes. Turbulent diffusion coefficient calculated numerically and measured with the MU radar are of $1-10 \mathrm{~m}^{2} / \mathrm{s}$ in different seasons in Shigaraki, Japan $\left(35^{\circ} \mathrm{N}\right.$, $\left.136^{\circ} \mathrm{E}\right)$. These values lead to the estimation of vertical ozone flux from the stratosphere to the troposphere of $(1-10) \times 10^{14}$, which may substantially add to the usually supposed ozone downward transport with the general atmospheric circulation. Therefore, local enhancements of IGW intensity and turbulence at tropospheric altitudes over mountains due to their orographic excitation and due to other wave sources may lead to the changes in tropospheric and total ozone over different regions.
\end{abstract}

Key words. Meteorology and atmospheric dynamics (Turbulence; Waves and tides) - Atmospheric composition and structure (Middle atmosphere composition and chemistry)

\section{Introduction}

One of the important problems is the role of gravity waves and turbulence in diffusion of ozone and gas species in the tropo-stratosphere. It is supposed recently that the main mechanism of the transport of admixtures influencing the ozone layer between the troposphere and the stratosphere is the general circulation of the atmosphere, creating upward motions near the equator and downward motions at the middle and high latitudes (Holton, 1990). Additional ozone transport from the stratosphere to the troposphere could be produced by mesoscale and small-scale processes (Lamarque

Correspondence to: N. M. Gavrilov

(gavrilov@ pobox.spbu.ru) and Hess, 2003). Mesometeorological processes may produce intrusions of stratospheric ozone into the troposphere, which are frequently observed (Lamarque and Hess, 2003). Small-scale turbulence provides mixing of atmospheric gases and may produce turbulent ozone fluxes due to vertical gradients of the ozone mixing ratio (Pavelin et al., 2002; Whiteway et al., 2003). But despite intensive research during the last years, there are still many uncertainties and unknowns about the role which mesoscale and small-scale processes may play in the stratospheric-tropospheric exchange.

A boundary between the troposphere and the stratosphere is denoted as the tropopause. Usually it is located not far from the temperature minimum. There are several definitions of the tropopause based on temperature structure and dynamical features of the atmosphere (Dameris, 2003). The heights obtained with different tropopause definitions may be approximately equal (Birner et al., 2002), or sometimes they may differ up to several kilometers. Therefore, in general, we should say about a quite thick. One of the important features is a sharp increase in potential temperature and its vertical gradient in the tropopause region (Birner et al., 2002). This makes the mean temperature profile above the tropopause much more stable than below, which commonly assumes turbulence suppression above the tropopause.

On the other hand, experiments with radars, balloons and aircrafts (Pavelin et al., 2001; Pavelin and Whiteway, 2002; Luce et al., 2002) show strong internal gravity waves (IGWs) and unstable turbulized layers near and above the tropopause. Maxima of IGW activity and turbulent diffusivity near the tropopause were systematically observed during multiyear observations with Japanese Middle and Upper (MU) Atmosphere radar in Shigaraki (Fukao et al., 1994; Murayama et al., 1994; Kurosaki et al., 1996).

In this paper we analyze IGW theory to show that the sharp change in the vertical temperature gradient near the tropopause temperature minimum can make a sharp increase in the amplitudes of IGWs propagating upwards from the troposphere. This can lead to IGW breaking and to generation of stronger turbulence, which may make the tropopause more 


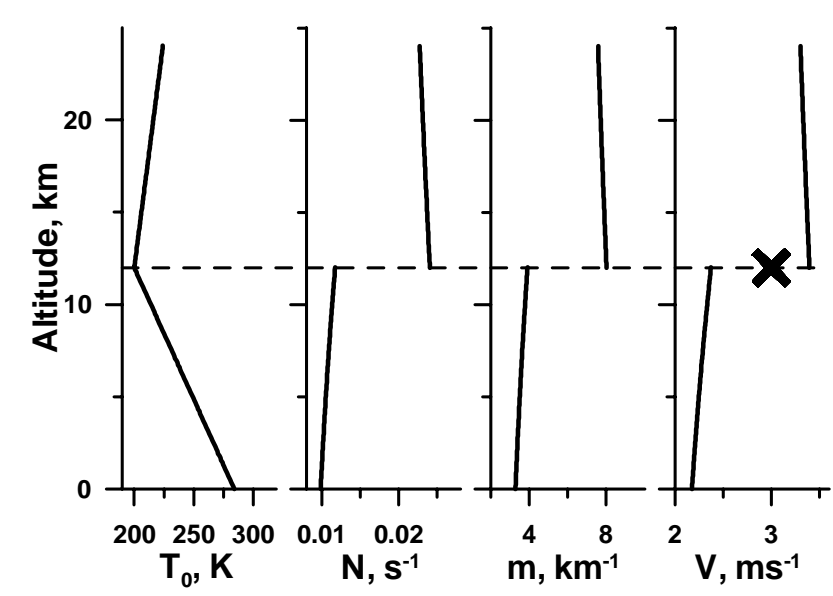

Fig. 1. Simple model of temperature profile, Brunt-Väisälä frequency, IGWs vertical wavenumber and corresponding sharp increase in amplitude of IGW with $\mathrm{c}=3 \mathrm{~m} / \mathrm{s}^{-1}$ (from left to right, respectively). Cross shows the IGW breaking level.

transparent for the diffusive transport of the admixtures between the troposphere and stratosphere.

We study this mechanism of IGWs and turbulence enhancing near the tropopause using a numerical model by Gavrilov and Fukao (1999), which describes IGW propagation and turbulence generation in the non-homogeneous atmosphere. The numerical model gives the integral energy characteristics of a spectrum of IGW harmonics with various frequencies, horizontal phase speeds and directions of propagation. The model includes IGW generation on the Earth's surface and inside the atmosphere, realistic vertical profiles of the mean wind and temperature, IGW dissipation, destruction of waves and generation of turbulence. The results of numerical calculations are compared with the measurements of parameters of IGWs and turbulence in the tropo-stratosphere with the MU radar of Japan.

\section{Numerical simulation of wave-induced turbulent dif- fusivity near the tropopause}

A quick increase in vertical temperature gradient at the tropopause may change the conditions of IGW propagation and may produce an increase in wave amplitudes. Therefore, IGWs propagating from the troposphere may break and produce stronger turbulence. This may cause stronger diffusion of atmospheric admixtures through the tropopause. Such diffusive transport of admixtures may make an addition to the traditionally assumed circulation with upward flux of atmospheric mass from the troposphere to the stratosphere in the equatorial region and its downward flux in the middle and high latitudes.

\subsection{Mechanism of IGW enhancement near the tropopause}

An increase in IGW amplitudes near the tropopause temperature minimum should be anticipated from the conservation of vertical wave flux of wave action. For low-frequency short IGWs having intrinsic frequency $\omega$ and vertical wave number $m$, satisfying to $\omega^{2} \ll N^{2}$ and $m^{2} \gg 1 / 4 H^{2}$ (where $N$ and $H$ are Brunt-Väisälä frequency and atmospheric scale heights, respectively), one can obtain the following expressions for vertical flux of wave action, $F_{a z}$ :

$F_{a z} \approx \rho_{0} V^{2} / 2 m ;|k / m| \approx \omega / N ; N^{2}=g\left(\partial T_{0} / \partial z+\gamma_{a}\right) / T_{0}$,

where $V$ and $k$ are the amplitude of horizontal velocity and horizontal wave number, respectively; $\rho_{0}$ and $T_{0}$ are the background atmospheric density and temperature; $g$ is accelerations due to gravity; $\gamma_{a}$ is the adiabatic temperature lapse rate. The tropopause is the region, where $\partial \mathrm{T}_{0} / \partial \mathrm{z}$ sharply increases from its substantial negative values in the troposphere below (Dameris, 2003). According to Eq. (1), an increase in $\partial \mathrm{T}_{0} / \partial \mathrm{z}$ gives a respective increase in $N^{2}$ and $|\mathrm{m}|$ at given values of $\omega$ and $k$. Due to the wave action conservation law (Andrews et al., 1987), $F_{a z}$ should be constant for nondissipative IGWs propagating in the atmosphere. Therefore, the increase in $|\mathrm{m}|$ near the tropopause should lead to the respective increase in $V^{2}$ in Eq. (1) for IGWs propagating from below.

Figure 1 represents a simple model of the tropopause composed of two pieces of linear temperature profiles with zero background wind. In this model the quantities $N, m$ and $V$ change their values abruptly at the level of temperature minimum (see Fig. 1). The increased IGW amplitude may become larger than the horizontal phase speed (cross in Fig. 1). In this case IGWs become unstable near the tropopause and generate turbulence due to wave breaking. Such strengthening of irregular wave and turbulent motions may produce increased diffusion and make the tropopause region more transparent for transport of atmospheric admixtures.

\subsection{Numerical model}

In this study, to evaluate the above mentioned possible mechanism of increasing IGW amplitudes and turbulence intensity near the tropopause, we use a numerical model of IGW propagation and turbulence generation in the atmosphere with realistic vertical profiles of background temperature and wind. The model was described by Gavrilov and Fukao (1999) and Gavrilov and Jacobi (2004). Therefore, we only make its short description here. The model assumes that the atmospheric wave fields can be represented by a spectrum of sinusoidal harmonics. The model calculates vertical distributions of parameters of a set of IGW harmonics representing a wave spectrum. In a stationary and horizontally homogeneous background atmosphere, the balance of the wave action is valid for each wave harmonic (see Gavrilov and Fukao, 1999; Gavrilov and Jacobi, 2004):

$\partial F_{a z} / \partial z=\rho_{0}\left(s V-N_{d} V^{2} / 2\right) / \omega$,

where $s$ is the strength of wave sources (see Gavrilov, 1997); $N_{d}$ is the rate of IGW dissipation. The main contributions to dissipation rate $N_{d}$ are from turbulent and molecular viscosity and heat conduction, radiative heat exchange, and ion drag (see Gavrilov, 1990). 
The parameter $s$ in Eq. (2) describes the strength of nonlinear wave sources of mass, momentum and heat in the atmosphere (see Gavrilov and Fukao, 1999). At low latitudes, substantial IGW emission may be produced by random convective motions (Alexander and Holton, 1997). Additional IGW generation may be provided by Lighthill-type nonlinear interactions of mesoscale meteorological motions (see Lighthill, 1952, 1978; Stein, 1967). Both convective and hydrodynamic IGW sources are supposed to be randomly distributed within the atmosphere. Gavrilov and Fukao (1999) supposed that each elementary wave source generates its own IGW with random observable frequency, $\sigma$, horizontal phase speed, $c$, and azimuth of propagation $\varphi$.

The wave harmonics from different sources produce a statistical ensemble of IGWs. Equation (2) may be solved for a selection of IGW harmonics with an arbitrary set of $\sigma_{i}, c_{j}$ and $\varphi_{k}$. Then, assuming a probability distribution function for the $s$ values, the average variances associated with the ensemble of IGW harmonics generated by random sources can be obtained. The strength of the wave sources $s$ in Eq. (2) can depend on $\sigma, \mathrm{c}$ and $\varphi$. While the atmospheric IGW spectrum is almost certainly not separable (Gardner, 1995), it is often not a bad approximation (Fritts and VanZandt, 1987), and in this simple model we suppose that

$s\left(\sigma, c, \varphi, v_{0}, N\right)=S\left(v_{0}, N\right) F_{\sigma}(\sigma) F_{c}(c) \Phi(\varphi)$,

where $v_{0}$ is the mean wind speed. The functions $F_{\sigma}(\sigma)$, $F_{c}(c)$ and $\Phi(\varphi)$ are used according to Gavrilov and Jacobi (2004). These functions are assumed to decrease at large $\sigma$, and small and large $c$, as might be expected for turbulent flows (Monin and Yaglom, 1971). The function $\Phi(\varphi)$ relates to the azimuth, $\varphi_{0}$, of the mean wind (see Gavrilov and Jacobi, 2004). The average variance of horizontal velocity produced by this ensemble may be calculated as described by Gavrilov and Fukao (1999) and Gavrilov and Jacobi (2004).

The main disadvantage of our numerical model is its dependence on altitude only. This means that the background fields and statistical characteristics of wave sources are assumed to be horizontally homogeneous. This assumption may not be valid for low-frequency IGWs, the energy of which propagates at extremely low elevation angles $\alpha \sim \omega / \mathrm{N} \ll 1$. IGWs with periods up to $1.5-2 \mathrm{~h}$ and substantial horizontal phase speeds propagate energy from near the surface to an altitude of $20 \mathrm{~km}$ within a horizontal distance of $\sim 300-400 \mathrm{~km}$. At such horizontal scales, climatological characteristics of the background wind, temperature and wave sources may be relatively uniform in many cases, and the numerical model described above may be used.

An important random IGW sources at low latitudes might be convective atmospheric motions (Alexander and Holton, 1997). At present, there are no adequate parameterizations of convectively generated IGWs. We might expect that the intensity of convective wave sources could depend on the mean Brunt-Väisälä frequency, which estimates a stability of the mean temperature profile influencing the conditions of convection development in the atmosphere.

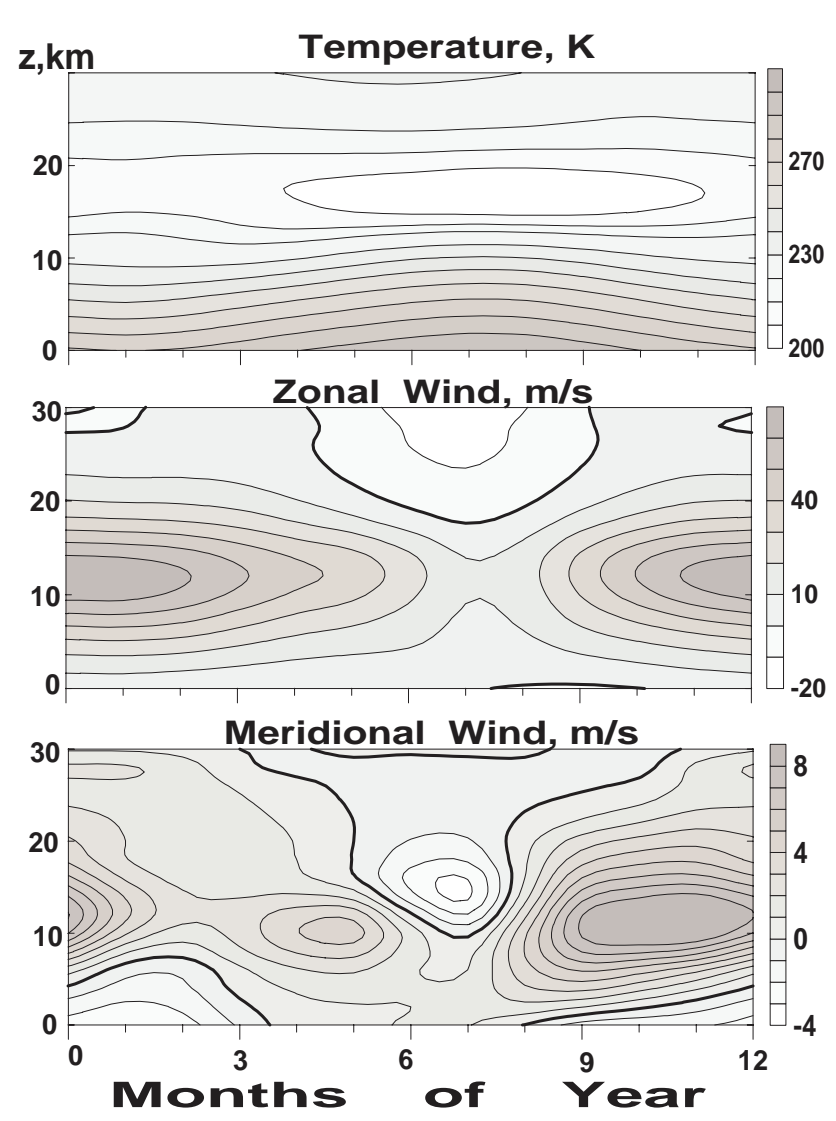

Fig. 2. Background temperature (top), and zonal (middle) and meridional (bottom) wind velocities at Shigaraki, Japan.

Additional IGW generation may be produced by mesoscale meteorological and irregular motions, which produce mesoscale turbulence in the atmosphere. The main contribution to these IGW nonlinear hydrodynamic sources comes from the nonlinear advective accelerations involved in the hydrodynamic momentum equation (see Drobyazko and Krasilnikov, 1985). Observations of the advective accelerations in the troposphere and stratosphere with the Japanese MU radar (see Gavrilov and Fukao, 2001) show their strong dependence on the mean wind velocity, $v_{0}$. Also, we may expect a dependence of $s$ on $N$, which may influence the inferred intensity of turbulent and convective motions in the atmosphere. Gavrilov and Fukao (1999) expressed $S\left(v_{0}, N\right)$ in Eq. (3) in the form of

$S\left(v_{0}, N\right)=S_{0} v_{0}^{n} / N^{q}$,

where $S_{0}, n$ and $q$ are constants. Equations (2)-(4) are solved here for a set of IGW harmonics representing a statistical ensemble of waves propagating from random IGW sources. The background wind components for altitudes 0 $30 \mathrm{~km}$ at Shigaraki, Japan, are presented in Fig. 2. We used the monthly mean temperatures and winds taken from the NCEP/NCAR Reanalysis database and averaged them over the years 1980-2000 and from the MU radar measurements as well (Murayama et al., 1994). 


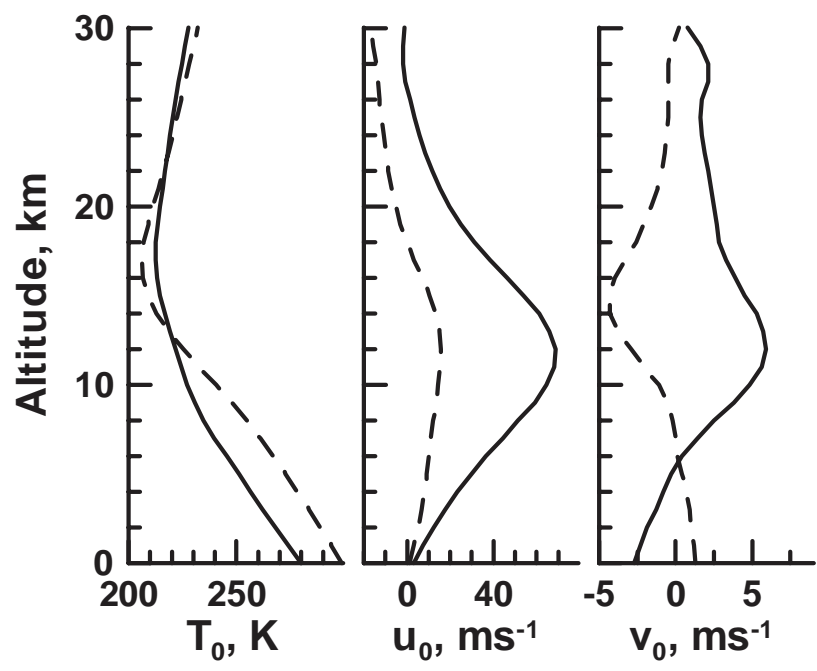

Fig. 3. Background profiles of temperature (left), zonal (middle) and meridional (right) wind velocities in January (solid lines) and July (dashed lines) at Shigaraki.

For altitudes above $30 \mathrm{~km}$ the background temperature and wind were taken from the MSISE-90 and HWM-93 models (Hedin, 1991; Hedin et al., 1996) for different months of the year. Equation (2) contains the rate of IGW dissipation $N_{d}$, due to turbulent and molecular viscosity and heat conduction, ion drag, and radiative heat exchange. These characteristics, as well as coefficients of turbulent viscosity and heat conduction and dissipation of IGW harmonics at critical and reflection levels, are calculated here, as described by Gavrilov and Fukao (1999). The influence of the critical layers leads, in the model, to stronger dissipation of IGW harmonics propagating in the direction of the mean wind and to the predominance of waves propagating in the opposite direction (Gavrilov, 1997). Such IGW filtering provides, in the model, the wave accelerations of the mean flow directed mainly opposite to the direction of the strato-mesospheric winds in the middle atmosphere (Gavrilov, 1997), which is consistent with recent views (Fritts and Alexander, 2003).

Except for IGW characteristics, the numerical model allows ones to estimate turbulent diffusivities caused by breaking IGWs. We use the method of calculating the turbulent diffusivities within zones of convective and dynamical instabilities caused by saturated and unsaturated IGWs developed by Gavrilov and Yudin (1992). The method is based on the closure of spectral turbulent equations using a semiempirical hypothesis for the spectral characteristics of turbulence.

The model has been validated previously by a comparison of the calculated average wave characteristics with the results of radar observations of the IGW climatology in the middle and upper atmosphere. The numerical model reproduces different types of seasonal variations of IGW intensity (see Gavrilov and Fukao, 1999), which have the winter maximum and summer minimum in the upper troposphere and stratosphere, and the solstice maxima and equinox minima in the mesosphere (see Murayama et al., 1994). Gavrilov et al. (2003) and Gavrilov and Jacobi (2004) applied the numerical model for the interpretation of seasonal variations of the wind and ionospheric drift velocity variances observed at altitudes of $70-110 \mathrm{~km}$ with MF radar at Hawaii and with the D1 reflection method at Collm, Germany. The model reproduces a transition from the solstice maxima of the velocity variances observed near $70-80 \mathrm{~km} \mathrm{~km}$ to the equinox maxima at higher altitudes.

\subsection{Results of numerical simulation}

In this study the numerical model involving Eqs. (2)-(4) was run for the background atmosphere representing different months of the year at the location of the MU radar (see Fig. 2). The numerical methods are further described by Gavrilov (1990). The vertical integration step is of $250 \mathrm{~m}$. The equations were solved for a set of $50 \times 50 \times 12$ IGW harmonics, where the multipliers denote the numbers of wave frequencies, horizontal phase speeds and azimuths, respectively. The IGW parameters cover the frequency ranges of $\sigma \sim 6 \times 10^{-4}-6 \times 10^{-3} \mathrm{rad} \mathrm{s}^{-1}$, horizontal phase speeds of c $\sim 3-100 \mathrm{~m} / \mathrm{s}^{-1}$ and azimuths of $\varphi \sim 0^{\circ}-360^{\circ}$. The first two grids were logarithmically spaced (proportional to $\ln \sigma$, and $\ln \mathrm{c}$ ) within the specified intervals. The values of the constants determining the spectral distributions of the wave sources in the model are the same as those used by Gavrilov and Jacobi (2004). Parameters of the model were chosen to provide IGW power spectral slopes of $\sigma^{-5 / 3}$ and $m^{-3}$ at large $\sigma$ and $m$, corresponding to some observations and theoretical studies (VanZandt, 1982). The parameters were chosen to give the best fit of the calculated results to observations (see below). Gavrilov and Fukao (1999) showed that this model can reproduce realistic seasonal variations of IGW intensity in the troposphere and mesosphere with $n=2$ in Eq. (4). A strong dependence of the intensity of wave sources on the mean wind in the tropo-stratosphere is confirmed by recent MU radar measurements of nonlinear advective accelerations (Gavrilov and Fukao, 2001). Therefore, in Eq. (4) we use the values of $n=2, S_{0}=10^{-5} \mathrm{~m}^{-1} \mathrm{~s}^{-2}$ and $q=2$.

In this study, we calculated vertical profiles of the turbulent diffusion coefficient produced by a spectrum of breaking IGWs. Specifically, we studied the influence of the sharp changes in the vertical temperature gradient in the changes of the conditions of IGW propagation, increasing their destruction and forming a maximum of the turbulent diffusivity near the tropopause. The mean profiles of wind and temperature are specified for the geographic coordinates of the observation site Shigaraki in Japan, from which we have the MU radar data. Figure 3 shows the vertical profiles of background temperature and wind in January and July.

Figure 4 represents the corresponding calculated standard deviations of wind velocity produced by a spectrum of IGWs in the ranges of periods $0.3-6 \mathrm{~h}$ and horizontal phase speeds $3-60 \mathrm{~m} / \mathrm{s}$ and of the coefficient of turbulent diffusion produced by breaking IGWs. One can see that the IGW intensity and the turbulent diffusivity have main maxima at alti- 


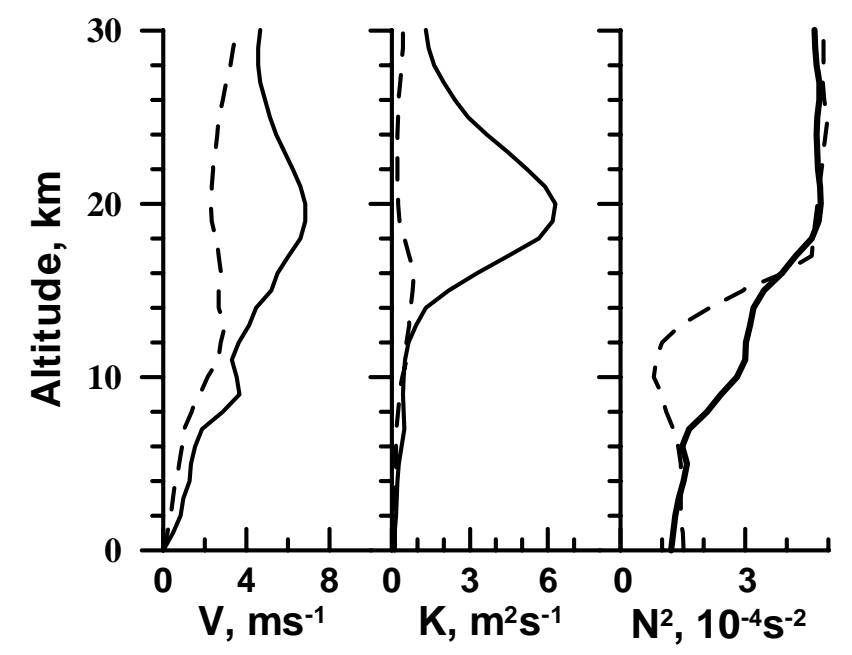

Fig. 4. Calculated vertical profiles of IGW horizontal velocity standard deviation (left), turbulent viscosity (middle) and Brunt-Väisälä frequency squared (right) for January (solid lines) and July (dashed lines) at Shigaraki.

tudes $15-20 \mathrm{~km}$, corresponding to the regions with a sharp increase, and maxima of temperature gradient and BruntVäisälä frequency in the right plot of Fig. 4. The January temperature profile has two regions of clear changes in vertical temperature gradient and Brunt-Väisälä frequency: at altitudes 9-10 km and 17-19 km (see Figs. 3 and 4). Therefore, one can see a local maximum of IGW amplitudes and turbulent viscosity at altitudes $9-10 \mathrm{~km}$ in the left plot of Fig. 3 . A reason for maxima of IGW amplitudes and turbulent viscosity in our model is mainly due to a sharp increase in the Brunt-Väisälä frequency in the tropopause region. This leads to an increase in IGW amplitudes (see Sect. 2.1), wave instability and generation of stronger turbulence in our model. The stronger turbulence increases IGW dissipation; therefore, wave momentum flux and amplitudes become smaller at higher altitudes in Fig. 4. This forms a layer of increased IGW amplitude and turbulent viscosity in the tropopause region in Fig. 4.

Calculated seasonal-altitude distributions of wind variances produced by the IGW ensemble are shown in Fig. 5 . One can see substantial seasonal variations with maximum values in winter and the minimum in summer. This maximum occurs just above the tropo-stratospheric jet stream and near the tropopause located at altitudes $15-18 \mathrm{~km}$, depending on the season in Fig. 2. Corresponding distributions of turbulent viscosities produced by breaking IGWs are shown in Fig. 6. They also have a maximum in winter and a minimum in summer at altitudes $15-20 \mathrm{~km}$. In summer, the maxima of IGW variances and turbulent viscosity in Figs. 4 and 5 are lower that those in winter. The measurements of IGW intensity and turbulent viscosity with the MU radar at Shigaraki also show their maxima near the tropopause (Murayama et al., 1994; Fukao et al., 1994) and with larger values in winter than in summer. Our calculated values correspond well

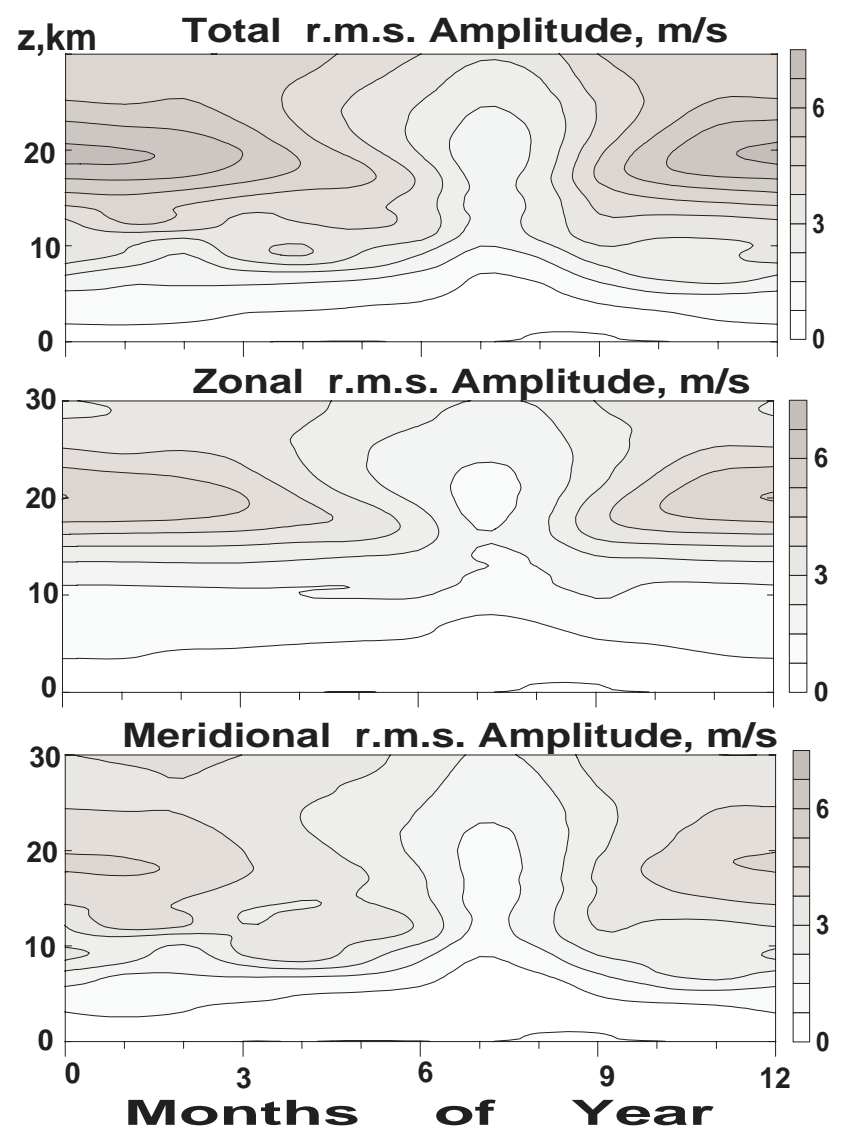

Fig. 5. Calculated seasonal distributions of total (top), zonal (middle) and meridional (bottom) wind standard deviations produced by IGW spectrum.

enough to the measured ones as further discussed in Sect. 3 below.

Our numerical model allows us to also calculate such important characteristics as wave momentum flux and acceleration of the mean flow produced by dissipating IGWs, which are shown in Fig. 6. These characteristics are important for estimating the IGW influence of atmospheric general circulation. One can see that the wave accelerations are mainly positive below the altitudes of $15-16 \mathrm{~km}$, and are negative above these altitudes. In our model, positive zonal wave accelerations are created by IGW harmonics propagating eastwards. They may dissipate at critical layers within tropostratospheric jet streams and lose their energy there. Negative zonal wave accelerations at larger altitudes are produced by IGWs propagating westward. They do not meet critical levels inside the tropo-stratospheric jet stream, have larger amplitudes, become unstable and produce larger turbulence and wave accelerations at higher altitudes, than the eastward propagating IGWs.

\subsection{Estimating vertical ozone fluxes}

The presence of turbulence in the atmosphere produces the mixing of atmospheric gases. 


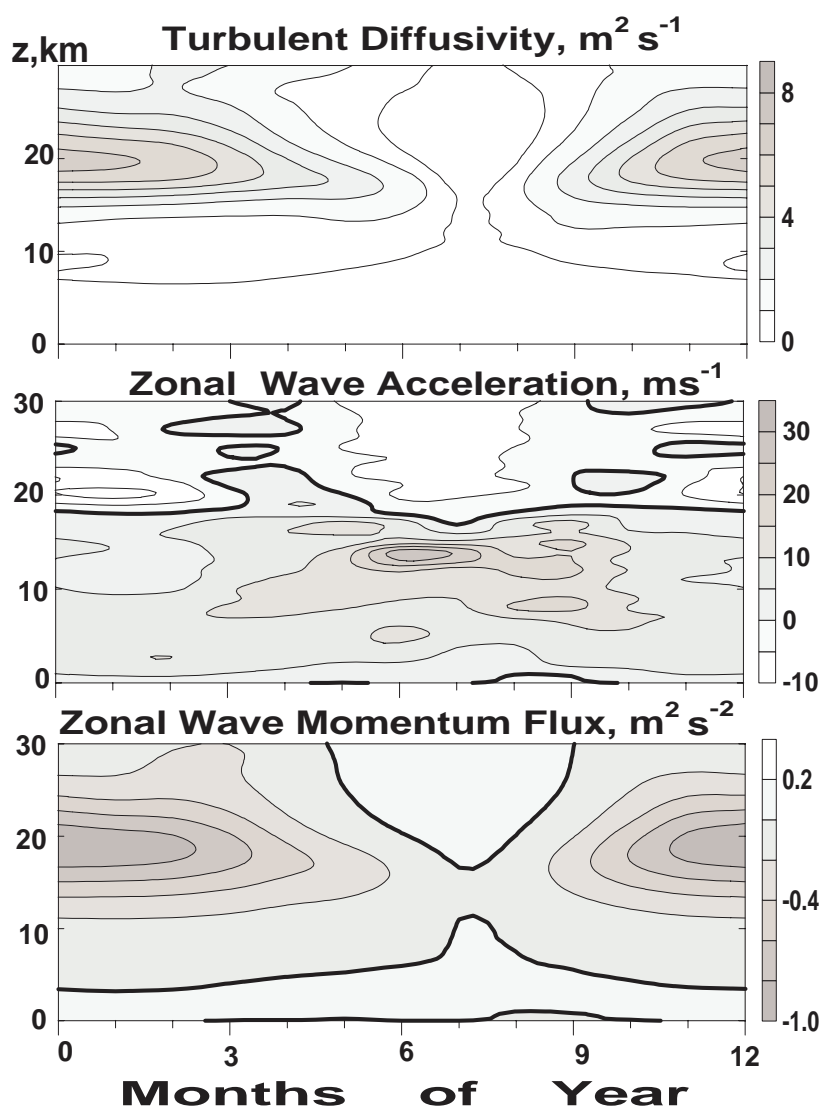

Fig. 6. Calculated distributions of turbulent viscosity (top), wave acceleration of the mean flow (middle) and vertical component of zonal wave momentum flux (bottom). Solid lines correspond to zero values.

Possible increase in IGW intensity and turbulence near the tropopause described above may lead, in particular, to an increase in fluxes of atmospheric admixtures from the troposphere to the stratosphere or back, depending on the vertical distribution of the particular admixture. In particular, turbulence may influence descending of ozone from the stratosphere to the troposphere. The vertical flux of an admixture can be described by the following formula:

$F=-K n c \partial(\ln c) / \partial z$,

where $K$ is the turbulent diffusion coefficient; $n$ is the number density of the atmosphere; $c$ is the mixing ratio of the considered admixture. Using the values of $c$ for the altitudes of $12-17 \mathrm{~km}$ from the model of vertical ozone profile (Zuev and Komarov, 1986) and the values of $K \sim 1-10 \mathrm{~m}^{2} / \mathrm{s}$ shown in Figs. 3 and 5, we may obtain the estimate $F \sim(1-$ 10) $\times 10^{14} \mathrm{~m}^{-2} \mathrm{~s}^{-1}$.

The main, recently assumed, global-scale mechanism of penetration of the stratospheric ozone into the troposphere is its transport by the general atmospheric circulation from equatorial latitudes and descending of the air in the middle latitudes (Holton, 1990). The mean downward ozone flux due to the circulation transport is estimated to be about $\mathrm{F}$ $\sim 7 \times 10^{14} \mathrm{~m}^{-2} \mathrm{~s}^{-1}$ (Ebel et al., 1993), while the estimations of tropospheric ozone require the fluxes of stratospheric ozone to be of the order of $(4-8) \times 10^{14} \mathrm{~m}^{-2} \mathrm{~s}^{-1}$ (e.g. Crutzen, 1988). Comparison of these values with the estimations of the ozone diffusion flux obtained above show that these values may become comparable. Therefore, in some regions and times turbulence generation by gravity waves near the tropopause may produce noticeable transport of ozone from the stratosphere to the troposphere.

One can estimate the characteristic time scale required for the transport of all stratospheric ozone into the troposphere (in the absence of its sources): $\tau \sim q / F$, where $q$ is the total ozone content in the atmospheric column above the altitude considered. The estimates of $K$ described above, together with the model of vertical ozone distribution (Zuev and Komarov, 1986), lead to the values of $\tau \sim 0,3-3$ days. These values show that the changes in IGW intensity and turbulence near the tropopause may lead, relatively fast, to the changes in the stratospheric ozone concentration and to a shift in the photochemical equilibrium.

\section{Comparison with measurements and discussion}

The numerical model used in this study is a simple, onedimension model of IGW propagation. More realistic threedimension simulations of turbulent mixing in the unstable jet streams near the tropopause (Joseph et al., 2003, 2004) also revealed maxima of turbulent characteristics in the regions with a sharp increase in the Brunt-Väisälä frequency in the tropopause region. This three-dimensional model does not include breaking IGWs and involves wind shears as the main source of turbulent energy inside the jet stream. Possible growing of IGW amplitudes and wave breaking discussed above may produce additional turbulence generation within and above the tropopause jet stream.

It is interesting to compare the results of numerical calculations described in the previous section with the results of the measurements with the MU radar operating in Shigaraki $\left(35^{\circ} \mathrm{N}, 136^{\circ} \mathrm{E}\right)$ since the year 1984 . Numerous studies of IGWs and turbulent diffusivity in the tropo-stratosphere were made with this radar (Fukao et al., 1994; Murayama et al., 1994; Kurosaki et al., 1996; etc.) These studies showed that the mean value and variance of wind velocity, as well as turbulent diffusivity, have strong seasonal variations near the tropopause, with the maxima in winter and minima in summer. The standard deviations of variations of zonal and meridional wind components with time scales of $5 \mathrm{~min}-$ $21 \mathrm{~h}$ are about $3.5-4 \mathrm{~m} / \mathrm{s}$ in January and $2-2.5 \mathrm{~m} / \mathrm{s}$ in July at heights of $15-17 \mathrm{~km}$ (Murayama et al., 1994). Measured mean vertical profiles of kinetic energy of wind variations with periods of $5 \mathrm{~min}-2 \mathrm{~h}$ have the main maxima at altitudes $11-13 \mathrm{~km}$ in winter and $13-14 \mathrm{~km}$ in summer (Murayama et al., 1994). They correspond to the locations of the maximum of tropo-stratospheric jet streams in different seasons. In many cases individual profiles of the kinetic energy have the secondary maxima at altitudes $15-20 \mathrm{~km}$ (Murayama et al., 1994). 
In comparing these experimental results with our numerical modelling one should keep in mind that the calculations of Sect. 3 refer to propagating IGWs, but several reasons for the wind variability in the tropo-stratosphere may occur. One of them may be the generation of turbulence due to wind shears inside the jet stream (Joseph et al., 2003, 2004). Another reason could be the generation of mesoscale irregularities by instabilities within the tropo-stratospheric, jet stream. These irregularities could be more intensive in winter, when the speed of the jet is higher over Shigaraki. Such irregularities are located mainly inside the jet stream, producing some sort of mesoscale turbulence, which could be much stronger, than propagating IGWs located there. Due to nonlinear interactions, this quasi-two-dimensional mesoscale turbulence may generate gravity waves, which can propagate upwards. Gavrilov et al. (1999) used 9-beam MU radar measurements for estimating mesoscale variability of nonlinear advective accelerations responsible for Lighthill-type IGW generation. They showed that these accelerations are largest at altitudes of the tropo-stratospheric jet stream maxima. IGWs generating by nonlinear interactions of this mesoscale turbulence inside the jet stream and propagating from other tropospheric sources could be the third reason for observed wind variability. These waves could be relatively weak compared with irregularities inside the tropo-stratospheric jet, but may become noticeable at higher altitudes, where their amplitudes grow. IGWs propagating from below may form secondary maxima of wind variability at altitudes $15-20 \mathrm{~km}$ observed in many individual monthly profiles by Murayama et al. (1994) and expected from the numerical modelling of Sect. 2.

The question is how well do these observed secondary maxima of wind variability correspond to the observed tropopause heights. Numerical simulation in Sect. 2 was made for climatological mean temperature and wind distributions, which are smooth with clear temperature minima. In real experiments the tropopause height is not very stable. Mesoscale disturbances and enhancing IGWs may superimpose to the mean temperature profile shifting the main temperature minimum and forming additional minima. One should expect that these dynamical disturbances of the tropopause could be larger in winter, when mesoscale disturbances within the jet stream and IGW activity are larger. In reality, Murayama et al. (1994) observed larger variability of the tropopause height over Shigaraki in winter. In many cases temperature minima appeared at altitudes $9-10 \mathrm{~km}$ within the tropo-stratospheric jet. Climatologically speaking, this corresponds to a clear change in the vertical temperature gradient at altitudes $9-10 \mathrm{~km}$ in January, in Fig. 3. Therefore, the maxima of propagating IGWs caused by a sharp increase in the Brunt-Väisälä frequency near such low temperature minima (see Fig. 4) may overlap with the wind variability produced by the jet streams instabilities. Also, substantial altitude variability of the tropopause creates difficulties in the localization of corresponding IGW maxima in winter.

In summer, the tropopause heights over Shigaraki are more stable and vary between 15 and $17 \mathrm{~km}$ (Murayama et al., 1994). As a result, many vertical profiles of kinetic energy of wind variations with periods of $5 \mathrm{~min}-2 \mathrm{~h}$ in Fig. 8 of the paper by Murayama et al. (1994) have secondary maxima at altitudes $17-19 \mathrm{~km}$, just above the tropopause. This broadens the maximum at the mean summer profile of the kinetic energy in the same figure, which expands from 13 up to $18 \mathrm{~km}$ (see Murayama et al., 1994). This shows that the increase in the Brunt-Väisälä frequency near the tropopause may really influence IGW propagation and may increase their amplitudes.

Fukao et al. (1994) studied seasonal variations of eddy diffusivity, $K$, measured by the MU radar, using the Doppler velocity spectral width method. They found broad distributions of $K(z)$ with maxima between altitudes $12-14 \mathrm{~km}$ and median values of $K>1 \mathrm{~m}^{2} / \mathrm{s}$ up to altitudes $16-20 \mathrm{~km}$, depending on season. The winter median values of $K$ are generally larger than those in summer, although high tropostratospheric jet velocity in winter produces large broadening of the Doppler velocity spectrum and makes measurements of $K$ almost impossible. Kurosaki et al. (1996) obtained annual mean vertical profiles of $K$ for the years 1986-1992. They found median values of $K$ up to $6 \mathrm{~m}^{2} / \mathrm{s}$ at altitudes 10 $14 \mathrm{~km}$ and $K>1 \mathrm{~m}^{2} / \mathrm{s}$ up to altitudes $17-18 \mathrm{~km}$. Therefore, the influence of the increase of the Brunt-Väisälä frequency on the increasing of IGW activity near the tropopause may slow down the decrease in $K$ above tropo-stratospheric jet stream and provides substantial turbulent diffusivities near the tropopause.

Gavrilov et al. (2004) estimated turbulent diffusivities, $K$, and vertical turbulent ozone fluxes, $F$, in the tropostratosphere from simultaneous measurements with the MU radar and ozonesondes at Shigaraki, Japan, in April 1998. They obtained local maximum values of $K$ up to 6$10 \mathrm{~m}^{2} / \mathrm{s}^{-1}$ and $F$ up to $-(30-40) \times 10^{14-2} \mathrm{~s}^{-1}$, at altitudes, $8-14 \mathrm{~km}$ in the tropopause region. Both $K$ and $F$ have complicated intermittent vertical structures with multiple local maxima and minima of $K$ and $F$. Therefore, further study is required for obtaining climatological mean values of $K$ and $F$ in different locations and seasons.

Pavelin et al. (2002) and Whiteway et al. (2003) reported about smaller values of $K \sim 1-2 \mathrm{~m}^{2} / \mathrm{s}^{-1}$ near the tropopause, which were measured with an aircraft over England. One reason for $K$ differences between Japan and Europe may be much larger speeds of the jet stream over Japan. According to Murayama et al. (1994) the speed of the jet stream over Shigaraki in January may reach up to $90-100 \mathrm{~m} / \mathrm{s}^{-1}$, which is much larger than the speeds of the jet streams observed over Europe (Pavelin et al., 2002; Whiteway et al., 2003). Also, the MU radar is located in the mountain region, while aircraft measurements of Pavelin et al. (2002) and Whiteway et al. (2003) were made over plane surface. Therefore, differences in orography and dynamical activity of the atmosphere may lead to local differences of IGW intensity, turbulence and ozone fluxes over different regions.

Another reason for the discrepancies between aircraft and MST radar measurements of $K$ may be the possible overestimation of $K$ measured by the radars. There are several reasons which may cause such an overestimation. 
One of them is the Doppler spectrum broadening caused by the influence of the mean wind and its vertical gradient (see Hocking, 1985). Other formulae for the Doppler spectral width correction for the broadening were developed by Nastrom (1997). VanZandt et al. (2002) developed a dualbeamwidth method for estimating the Doppler spectral width of MST radars. The authors showed that after correction their method may give smaller values of the spectral width than the one-beamwidth method used by Fukao et al. (1994) and Kurosaki et al. (1996).

Another problem for the MST radar measurements of turbulence is connected with the formulae used for estimating effective turbulent diffusivity from measured Doppler spectrum width. Hocking (1999) emphasised the role of spatial and temporal intermittency, which may be produced by IGWs creating regions of instability separated by regions of stability. Dewan (1981) and Woodman and Rastogi (1984) suggested that the random occurrence of turbulent layers may produce a random process of intermittent diffusion and an effective turbulent diffusivity of this ensemble should be introduced. Fritts and Dunkerton (1985) and Gavrilov and Yudin (1992) showed that the intermittency may lead to the difference in diffusivity of momentum and heat, causing an increase in the effective Prandtl number from 1 up to 3 . High resolution balloon temperature measurements showed that turbulence in the tropo-stratosphere most frequently occur within relatively thin unstable layers (Luce et al., 2002). Therefore, turbulent transport of a particle may occurs only within this layer until it dies out. Then the particle remains nearly stationary because of negligible molecular diffusion. Later in time, another turbulent layer forms around the particle and further transport over the depth of the layer is possible. Therefore, climatologically, speaking, the transport of atmospheric species may depend on an effective diffusivity averaged over substantial time intervals rather than on local turbulent diffusivities measured at a particular experiment. Taking account of this as well as other aspects of the problem, Hocking (1999) supposed that the relation between turbulent diffusivity and energy dissipation rate measured by MST radars may not be so simple as it is usually assumed.

Resulting effective diffusivity caused by random intermittent diffusion might be smaller than local diffusivities measured by radar within turbulent regions. Estimations of vertical ozone fluxes from the stratosphere to the troposphere are made in Sect. 2.4 for values of $K \sim 1-10 \mathrm{~m}^{2} / \mathrm{s}$ observed with the MU radar. A comparison of these estimations with the fluxes caused by atmospheric general circulation (see Sect. 2.4) shows that even for values of $K$ up to several times smaller than those used in Sect. 2.4, turbulent ozone flux could be a noticeable addition to conventional transport of ozone due to circulation from the tropics to the middle latitudes. Therefore, improvement of the methods of MST radar studies of atmospheric turbulence and atmospheric admixtures transport through the tropopause are very urgent and important.

Estimations of the time scales of stratospheric ozone changes due to turbulent diffusion, $\tau$, in Sect. 2.4, show that the variability of turbulence activity near the tropopause due to meteorological processes and local conditions may result in noticeable changes in stratospheric, tropospheric and total ozone. Satellite observations show total ozone variability related to tropical cyclones (Nerushev, 1996). One of the mechanisms for such relations could be an increase in the instability and turbulence, within a cyclone, which leads to more active ozone transport from the stratosphere into the troposphere. Local enhancements of IGW intensity and turbulence due to their orographic excitation, may lead to the changes in total ozone over the mountain regions. This may explain, for example, the observed anomalies of total ozone over the mountain regions (Kazimirovsky and Matafonov, 1998).

\section{Conclusions}

A numerical simulation performed in this paper shows that a sharp change in the vertical temperature gradient and the Brunt-Väisälä frequency near the tropopause may produce an increase in amplitudes of IGWs propagating upward from the troposphere, wave breaking and generation of stronger turbulence. This may make the middle latitude tropopause more transparent for the transport of admixtures between the troposphere and the stratosphere. An increase in the turbulent diffusivities at altitudes near and above the tropopause is usually observed with the MU radar in Shigaraki, Japan $\left(35^{\circ} \mathrm{N}, 136^{\circ} \mathrm{E}\right)$. Turbulent diffusivity calculated numerically and measured with the MU radar is of $1-10 \mathrm{~m}^{2} / \mathrm{s}$ in the tropopause region in different seasons. This leads to the estimation of vertical ozone flux from the stratosphere to the troposphere of the order of $(1-10) \times 10^{14} \mathrm{~m}^{-2} / \mathrm{s}^{-1}$, which may become comparable with ozone downward fluxes with the general atmospheric circulation. Therefore, local enhancements of IGW intensity and turbulence at tropospheric altitudes over the mountains, due to their orographic excitation and due to other wave sources, may lead to the changes in the tropospheric ozone and the total ozone over different regions. Improving methods of MST radar evaluations of turbulent diffusivity and simultaneous ozonosonde measurements are highly important for better estimating the ozone turbulent transport through the tropopause.

Acknowledgements. This study was partly supported by the Russian Basic Research Foundation and by the International Science and Technology Center. The MU radar belongs to and is operated by the Center for Atmospheric and Space Research of Kyoto University.

Topical Editor U.-P. Hoppe thanks S. J. Reid and another referee for their help in evaluating this paper.

\section{References}

Alexander, M. J. and Holton, J. R.: A model study of zonal forcing in the equatorial stratosphere by convectively induced gravity waves, J. Atmos. Sci., 54, 408-419, 1997. 
Andrews, D. G., Holton, J. R., and Leovy, C. B.: Middle atmosphere dynamics, Academic Press, New York, 1987.

Birner, T., Dornbrack, A., and Schuman, U.: How sharp is the tropopause at midlatitudes?, Geophys. Res. Lett., 29(14), doi10.1029/2002GL015142, 2002.

Crutzen, P. J.: Tropospheric ozone: An overview, In I. S. A Isaken (ed.), Tropospheric Ozone, D. Reidel Publ. Company, Dordrecht, 3-32, 1988.

Dameris, M.: Tropopause, Enciclopedia of Atmospheric Sciences, Ed. by J. R. Holton, Academic Press, Amsterdam-New YorkTokyo, 2346-2348, 2003.

Dewan, E. M.: Turbulent vertical transport due to thin intermitten mixing layers in the atmosphere and other stable fluids, Science, 211, 1041-1042, 1981.

Drobyazko, I. N. and Krasilnikov, V. N.: Acoustic-gravity wave generation by atmospheric turbulence, Radiophysics, Izvestia VUZov of USSR, 28, 1357-1365, 1985.

Ebel, A., Elbern, H., and Oberreuter, A.: Stratosphere-troposphere air mass exchange and cross-tropopause fluxes of ozone. In Coupling processes in the lower and middle atmosphere, eds. Thrane, E. V. et al., Kluner, Dordrecht, 49-65, 1993.

Fritts, D. C. and Dunkerton, T. J.: Fluxes of heat and constituents due to convectively unstable gravity waves, J. Atmos. Sci., 42, 549-556, 1985.

Fritts, D. C. and VanZandt, T. E.: Effects of Doppler shifting on the frequency spectra of atmospheric gravity waves, J. Geophys. Res., 92, 9723-9732, 1987.

Fritts, D. C. and Alexander, M. J.: A review of gravity wave dynamics and effects in the middle atmosphere, Rev. Geophys., 41, 2003.

Fukao, S., Yamanaka, M. D., Ao, N., Hocking, W. K., Sato, T., Yamamoto, M., Nakamura, T., Tsuda, T., and Kato, S.: Seasonal variability of vertical eddy diffusivity in the middle atmosphere, 1. Tree-year observations by the middle and upper atmosphere radar, J. Geophys. Res., 99, 18 973-18 987, 1994.

Gardner, C. S., Tao, X., and Papen, G. C.: Simultaneous lidar observations of vertical wind, temperature and density profiles in the upper mesosphere: Evidence for nonseparability of atmospheric perturbation spectra, Geophys. Res. Lett., 22, 2877-2880, 1995.

Gavrilov, N. M.: Parameterization of accelerations and heat flux divergences produced by internal gravity waves in the middle atmosphere. J. Atmos. Terr. Phys., 52, 707-713, 1990.

Gavrilov, N. M.: Parameterization of momentum and energy depositions from gravity waves generated by tropospheric hydrodynamic sources. Ann. Geophys., 15, 1570-1580, 1997.

Gavrilov, N. M. and Fukao, S.: A comparison of seasonal variations of gravity wave intensity observed with the middle and upper atmosphere radar with a theoretical model. J. Atmos. Sci., 56, 3485-3494, 1999.

Gavrilov, N. M. and Fukao, S.: Hydrodynamic tropospheric wave sources and their role in gravity wave climatology of the upper atmosphere from the MU radar observations, J. Atmos. SolarTerr. Phys., 63, 931-943, 2001.

Gavrilov, N. M., Fukao, S., and Hashiguchi, H.: Multi-beam MU radar measurements of advective accelerations in the atmosphere, Geophys. Res. Lett., 26, 315-318, 1999.

Gavrilov N. M., Fukao, S., Hashiguchi, H., Kita, K., Sato, K., Tomikawa, Y. and Fujiwara, M.: Study of Atmospheric Ozone and Turbulence From Combined MU Radar and Ozonesonde Measurements in Shigaraki, Japan., Proc. XX Quadrennial Ozone Symp., Kos, Greece, 1-8 June, 41-42, 2004.

Gavrilov, N. M. and Jacobi, Ch.: A study of seasonal variations of gravity wave intensity in the lower thermosphere using LF D1 wind observations and a numerical model, Ann. Geophys., 22, 35-45, 2004.

Gavrilov, N. M., Riggin, D. M., and Fritts, D. C.: Mediumfrequency radar studies of gravity-wave seasonal variations over Hawaii $\left(22^{\circ} \mathrm{N}, 160^{\circ} \mathrm{W}\right)$, J. Geophys. Res., 108, 4655, 10.1029/2002JD003131, 2003.

Gavrilov, N. M. and Yudin, V. A.: Model for Coefficients of Turbulence and Effective Prandtl Number Produced by Breaking Gravity Waves in the upper Atmosphere, J. Geophys. Res., 97, 76197624, 1992.

Hedin, A. E.: Neutral atmosphere empirical model from the surface to lower exosphere MSISE-90, Extension of the MSIS thermosphere model into the middle and lower atmosphere, J. Geophys. Res., 96, 1159-1172, 1991.

Hedin, A. E., Fleming, E. L., Manson, A. H., Schmidlin, F. J., Avery, S. K., Clark, R. R., Franke, S. J., Fraser, G. J., Tsuda, T., Vial, F., and Vincent, R. A.: Empirical model for the upper, middle and lower atmosphere, J. Atmos. Terr. Phys., 58, 1421-1447, 1996.

Hocking, W. K.: Measurements of turbulent energy dissipation rate in the middle atmosphere by radar techniques: a review, Radio Sci., 20, 1403-1422, 1985.

Hocking, W. K.: The dynamical parameters of turbulence theory as they apply to middle atmosphere studies, Earth Planet. Sci., 51, 525-541, 1999.

Holton, J. R.: On the global exchange of mass between the stratosphere and troposphere, J. Atmos. Sci., 47, 392-395, 1990.

Joseph, B., Mahalov, A., Nikolaenko, B., and Tse, K. L.: High resolution DNS of jet stream generated tropopausal turbulence, Geophys. Res. Lett., 30, 1525, doi:10.1029/2003GL017252, 2003.

Joseph, B., Mahalov, A., Nikolaenko, B., and Tse, K. L.: Variability of turbulence and its outer scales in a model tropopause jet, J. Atmos. Sci., 61, 621-643, 2004.

Kazimirovsky, E. S. and Matafonov G. K.: Continental scale and orographic structures in the global distribution of the total ozone, J. Atmos. Solar-Terr. Phys., 60, 993-996, 1998.

Kurosaki, S., Yamanaka, M. D., Hashiguchi, H., Sato, T, and Fukao, S.: Vertical eddy diffusivity in the lower and middle atmosphere: a climatology based on the MU radar observations during 19861992, J. Atmos. Terr. Phys., 58, 727-734, 1996.

Lamarque J. F. and P. Hess. Stratosphere-troposphere exchange: Local processes, Enciclopedia of Atmospheric Sciences, Ed. By. J. R. Holton, Academic Press, Amsterdam - New York - Tokyo, 2143-2150, 2003.

Lighthill, M. J.: On sound generated aerodynamically, 1. General theory, Proc. Roy. Soc. London, A211, 564-587, 1952.

Lighthill, M. J.: Waves in fluids, Cambridge Univ. Press, 1978.

Luce, H., Fukao, S., Dalaudier, F., and Crochet, M.: Strong Mixing Events Observed near the Tropopause with the MU Radar and High-Resolution Balloon Techniques, J. Atmos. Sci., 59, 2885 2896, 2002.

Monin, A. S. and Yaglom, A. M.: Statistical fluid mechanics, 1, MIT Press, Cambridge, MA. 1971

Murayama, Y., Tsuda, T., and Fukao, S.: Seasonal variations of gravity wave activity in the lower atmosphere observed with the MU radar, J. Geophys. Res., 99, 23 057-23 069, 1994.

Nastrom, G. D.: Doppler radar spectral width broadening due to beamwidth and wind shear, Ann. Geophys., 15, 786-796, 1997.

Nerushev, A. F.: Tropical cyclone influence on the ozonosphere, Izv. Acad. Sci. USSR Atmos. Oceanic Phys., 31, 40-46, 1996.

Pavelin, E., Whiteway, J. A., and Vaughan, G.: Observation of grav- 
ity wave generation and breaking in the lowermost stratosphere, J. Geophys. Res., 106, 5173-5179, 2001.

Pavelin, E., Whiteway, J., Busen, R., and Hacker, J.: Airborne observations of turbulence, mixing and gravity waves in the tropopause region: J. Geophys. Res., 107(D10), 4084, doi:10.1029/2001JD000775, 2002a.

Pavelin, E. and Whiteway, J.: Gravity wave interactions around the jet stream, Geophys. Res. Lett., 29(21), 2024, doi:10.1029/2002GL015783, 2002.

Stein, R. S.: Generation of acoustic and gravity waves by turbulence in an isothermal stratified atmosphere, Solar Phys., 2, 285-432, 1967.

VanZandt, T. E.: A universal spectrum of buoyancy waves in the atmosphere, Geophys. Res. Lett., 9, 575-578, 1982.
VanZandt, T. E., Nastrom, G. D., Furumoto, J., Tsuda, T., and Clark, W. L.: A dual-beamwidth radar method for measuring atmospheric turbulent kinetic energy, Geophys. Res. Lett., 29, 1572, 10.1029/2001GL014283, 2002.

Whiteway, J. A., Pavelin, E. G., Busen, R., Hacker, J., and Vosper, S.: Airborn measurements of gravity wave breaking at the tropopause, Geophys. Res. Lett., 30(20), doi:10.1029/2003GL018207, 2003.

Woodman, R. F. and Rastogi P. K.: Evaluation of effective eddy diffusive coefficients using radar observations of turbulence in the stratosphere, Geophys. Res. Lett., 11, 243-246, 1984.

Zuev, V. E. and Komarov V. S.: Statistical models of temperature and gas components of the atmosphere, Hydrometeoizdat Press, Leningrad, 1986. 